Теорія Ймовір. та Матем. Статист. Вип. 78, 2008
Theor. Probability and Math. Statist.

No. 78, 2009, Pages 83-95

S 0094-9000(09)00764-9

Article electronically published on August 4, 2009

\title{
CONDITIONS FOR THE UNIFORM CONVERGENCE OF EXPANSIONS OF $\varphi$-SUB-GAUSSIAN STOCHASTIC PROCESSES IN FUNCTION SYSTEMS GENERATED BY WAVELETS
} UDC 519.21

\author{
YU. V. KOZACHENKO AND E. V. TURCHIN
}

\begin{abstract}
The expansions with uncorrelated coefficients in function systems generated by wavelets are constructed in the paper for second order stochastic processes. Conditions for the uniform convergence with probability one on a finite interval are found for expansions whose coefficients are independent. Conditions for the uniform convergence in probability on a finite interval are found for expansions of strictly $\varphi$-sub-Gaussian stochastic processes.
\end{abstract}

\section{INTRODUCTION}

The uniform convergence of random series with probability one or in probability are considered in [2, 7, 9, 17] and in some other papers.

The expansions of $\varphi$-sub-Gaussian stochastic processes with uncorrelated coefficients are obtained in this paper for function systems generated by wavelets. We find conditions for the uniform convergence on a finite interval with probability one for expansions with independent coefficients and those for the convergence in probability for expansions of strictly $\varphi$-sub-Gaussian stochastic processes.

The paper is organized as follows. Section 2 contains the main notions of the theory of strictly $\varphi$-sub-Gaussian random variables and stochastic processes. A theorem on the expansions of centered second order stochastic processes in series with uncorrelated coefficients for function systems generated by wavelet systems is stated in Section 3 . In Section 4. conditions for the uniform convergence with probability one on a finite interval are given for the expansions with independent terms. In Section 5 conditions are found for the uniform convergence in probability on a finite interval of expansions of strictly $\varphi$-sub-Gaussian stochastic processes.

All the results obtained in the paper are new.

\section{Strictly $\varphi$-Sub-Gaussian stochastic PROCESSES}

Definition 2.1 ([15]). A continuous even convex function $\varphi=\{\varphi(x), x \in \mathbf{R}\}$ is called an Orlicz $N$-function if $\varphi(0)=0, \varphi(x)>0$ for $x \neq 0$, and

$$
\lim _{x \rightarrow 0} \frac{\varphi(x)}{x}=0, \quad \lim _{x \rightarrow \infty} \frac{\varphi(x)}{x}=\infty .
$$

2000 Mathematics Subject Classification. Primary 60G07; Secondary 42C40.

Key words and phrases. Wavelets, $\varphi$-sub-Gaussian stochastic processes. 
Definition 2.2 ([5]). We say that condition $\mathbf{Q}$ holds for an $N$-function $\varphi$ if

$$
\lim _{x \rightarrow 0} \inf \frac{\varphi(x)}{x^{2}}=c>0
$$

(the case of $c=+\infty$ is not excluded).

The following definition of a $\varphi$-sub-Gaussian random variable is introduced in [5] and is a slight modification of the corresponding definition in [2].

Definition 2.3. Let $\{\Omega, \mathfrak{F}, \mathrm{P}\}$ be a standard probability space. Let condition $\mathbf{Q}$ hold for an $N$-function $\varphi$. A random variable $\xi$ is called $\varphi$-sub-Gaussian if

1) $\mathrm{E} \xi=0$,

2) the exponential moment $\mathrm{E} \exp \{\lambda \xi\}$ exists for all $\lambda \in \mathbf{R}$,

3) there exists a constant $a>0$ such that the inequality

$$
\operatorname{E} \exp \{\lambda \xi\} \leq \exp \{\varphi(\lambda a)\}
$$

holds for all $\lambda \in \mathbf{R}$.

The collection of $\varphi$-sub-Gaussian random variables is denoted by $\operatorname{Sub}_{\varphi}(\Omega)$. Note that $\operatorname{Sub}_{\varphi}(\Omega)$ is a Banach space with respect to the norm

$$
\tau_{\varphi}(\xi)=\sup _{\lambda \neq 0} \frac{\varphi^{(-1)}(\ln \mathrm{E} \exp \{\lambda \xi\})}{|\lambda|}
$$

(see [2, 5]). Examples of $\varphi$-sub-Gaussian random variables can be found in [5, 2. Notice

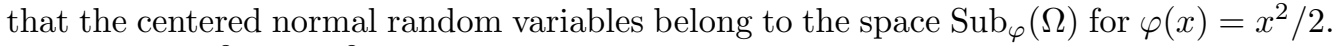
In this case, $\tau^{2}(\xi)=\sigma^{2}$.

Definition $2.4([8])$. A family $\Delta$ of random variables $\xi \in \operatorname{Sub}_{\varphi}(\Omega)$ is called strictly $\varphi$-sub-Gaussian if there exists a constant $C_{\Delta}>0$ such that

$$
\tau_{\varphi}^{2}\left(\sum_{i \in I} \lambda_{i} \xi_{i}\right) \leq C_{\Delta}^{2} \mathrm{E}\left(\sum_{i \in I} \lambda_{i} \xi_{i}\right)^{2}
$$

for an arbitrary finite collection of random variables $\xi_{i} \in \Delta$ and all $\lambda_{i} \in \mathbf{R}, i \in I$. The constant $C_{\Delta}$ is called the determining constant of the family $\Delta$.

Lemma 2.1 (8). The linear closure in the space $L_{2}(\Omega)$ of a strictly $\varphi$-sub-Gaussian family $\Delta$ is a strictly $\varphi$-sub-Gaussian family with the same determining constant.

Definition 2.5 (2]). A stochastic process $X=\{X(t), t \in T\}$ is called $\varphi$-sub-Gaussian if all random variables $X(t), t \in T$, are $\varphi$-sub-Gaussian.

Definition 2.6 ([8]). A stochastic process $X=\{X(t), t \in T\}$ is called strictly $\varphi$-subGaussian if the family of random variables $\{X(t), t \in T\}$ is strictly $\varphi$-sub-Gaussian with the determining constant $C_{T}$. The constant $C_{T}$ is called the determining constant of the process $X$.

Examples of $\varphi$-sub-Gaussian stochastic processes can be found in the book 2 and in the paper [5]. Examples of strictly $\varphi$-sub-Gaussian random variables and strictly $\varphi$-subGaussian stochastic processes are given in [8]. Note that a normal centered stochastic process is $\varphi$-sub-Gaussian with $\varphi(x)=x^{2} / 2$ and with the determining constant $C_{T}=1$. 


\section{ExpANSIONS OF STOCHASTIC PROCESSES IN SERIES WITH UNCORRELATED COEFFICIENTS}

Let $\phi=\{\phi(x), x \in \mathbf{R}\}$ be a function of the space $L_{2}(\mathbf{R})$ and let $\widehat{\phi}(y)$ denote the Fourier transform of $\phi$ :

$$
\widehat{\phi}(y)=\int_{\mathbf{R}} e^{-i y x} \phi(x) d x .
$$

A function $\phi$ is called an $f$-wavelet $([6$, [14) if

1) the function system $\{\phi(x-k), k \in \mathbf{Z}\}$ is orthonormal in the space $L_{2}(\mathbf{R})$;

2) there exists a periodic function $m_{0}(y)$ with period $2 \pi$ such that $m_{0} \in L_{2}(0,2 \pi)$ and almost everywhere

$$
\widehat{\phi}(2 y)=m_{0}(y) \widehat{\phi}(y)
$$

3) $\widehat{\phi}(0) \neq 0$ and $\widehat{\phi}(y)$ is continuous at zero.

Define the function $\widehat{\psi}(y)$ as follows:

$$
\widehat{\psi}(y)=\overline{m_{0}\left(\frac{y}{2}+\pi\right)} \exp \left\{-i \frac{y}{2}\right\} \widehat{\phi}\left(\frac{y}{2}\right) .
$$

Let $\psi(x)$ be the inverse Fourier transform of the function $\widehat{\psi}(y)$, that is,

$$
\psi(x)=\frac{1}{2 \pi} \int_{\mathbf{R}} e^{i y x} \widehat{\psi}(y) d y .
$$

A function $\psi(x)$ is called the $m$-wavelet corresponding to the $f$-wavelet $\phi$.

Put

$$
\phi_{j k}(x)=2^{j / 2} \phi\left(2^{j} x-k\right), \quad \psi_{j k}(x)=2^{j / 2} \psi\left(2^{j} x-k\right), \quad j \in \mathbf{Z}, k \in \mathbf{Z} .
$$

It is known that $\left\{\phi_{0 k}, \psi_{j k}, j=0, \ldots, \infty, k \in \mathbf{Z}\right\}$ is an orthonormal complete system in $L_{2}(\mathbf{R})($ see $[3,6])$.

Theorem 3.1. Let $X=\{X(t), t \in \mathbf{R}\}$ be a centered stochastic process such that $\mathrm{E}|X(t)|^{2}<\infty$ for all $t \in \mathbf{R}$ and let

$$
R(t, s)=\mathrm{E} X(t) \overline{X(s)}
$$

Assume that $\phi=\{\phi(\lambda), \lambda \in \mathbf{R}\}$ is an $f$-wavelet and $\psi=\{\psi(\lambda), \lambda \in \mathbf{R}\}$ is the $m$-wavelet corresponding to $\phi$. Denote

$$
\phi_{j k}(x)=2^{j / 2} \phi\left(2^{j} x-k\right), \quad \psi_{j k}(x)=2^{j / 2} \psi\left(2^{j} x-k\right), \quad j \in \mathbf{Z}, k \in \mathbf{Z} .
$$

Assume that $R(t, s)$ is represented in the form

$$
R(t, s)=\int_{\mathbf{R}} u(t, \lambda) \overline{u(s, \lambda)} d \lambda,
$$

where $u(t, \lambda)$, as a function of $\lambda \in \mathbf{R}$, is Borel for all $t \in \mathbf{R}$ and such that

$$
\int_{\mathbf{R}}|u(t, \lambda)|^{2} d \lambda<\infty
$$

for all $t \in \mathbf{R}$. Then

$$
X(t)=\sum_{k \in \mathbf{Z}} \xi_{0 k} a_{0 k}(t)+\sum_{j=0}^{\infty} \sum_{k \in \mathbf{Z}} \eta_{j k} b_{j k}(t),
$$


where the series converges for all $t \in \mathbf{R}$ in the mean square sense, that is,

$$
\begin{gathered}
\sum_{k \in \mathbf{Z}}\left|a_{0 k}(t)\right|^{2}+\sum_{j=0}^{\infty} \sum_{k \in \mathbf{Z}}\left|b_{j k}(t)\right|^{2}<\infty \\
a_{0 k}(t)=\frac{1}{\sqrt{2 \pi}} \int_{\mathbf{R}} u(t, y) \overline{\widehat{\phi}_{0 k}(y)} d y=\frac{1}{\sqrt{2 \pi}} \int_{\mathbf{R}} u(t, y) \overline{\widehat{\phi}(y)} e^{i k y} d y, \\
b_{j k}(t)=\frac{1}{\sqrt{2 \pi}} \int_{\mathbf{R}} u(t, y) \overline{\widehat{\psi}_{j k}(y)} d y=\frac{1}{\sqrt{2 \pi}} \int_{\mathbf{R}} u(t, y) 2^{-j / 2} \exp \left\{i \frac{y}{2^{j}} k\right\} \overline{\widehat{\psi}\left(\frac{y}{2^{j}}\right)} d y,
\end{gathered}
$$

$\widehat{\phi}_{0 k}(y)$ and $\widehat{\psi}_{j k}(y)$ are the Fourier transforms of the functions $\phi_{0 k}(x)$ and $\psi_{j k}(x)$, respectively, $\xi_{0 k}$ and $\eta_{j k}$ are centered random variables such that

$$
\mathrm{E} \xi_{0 k} \overline{\xi_{0 l}}=\delta_{k l}, \quad \mathrm{E} \eta_{m k} \overline{\eta_{n l}}=\delta_{m n} \delta_{k l}, \quad \mathrm{E} \xi_{0 k} \overline{\eta_{n l}}=0
$$

and $\delta_{k l}$ is the Kronecker symbol.

Remark 3.1. Let $H_{X}$ be the mean square closure of the sums

$$
\sum_{k=1}^{m} c_{k} X\left(t_{k}\right), \quad t_{k} \in \mathbf{R}
$$

and let $H_{\xi, \eta}$ be the mean square closure of the sums

$$
\sum_{j=1}^{m} \sum_{k=1}^{n} c_{k j} \eta_{k j}+\sum_{k=1}^{s} b_{k} \xi_{0 k}
$$

If the system $\{u(t, \lambda)\}$ is complete (in the sense that if $\int_{\mathbf{R}} u(t, \lambda) \overline{f(\lambda)} d \lambda=0$ for some function $f \in L_{2}(\mathbf{R})$ and all $t \in \mathbf{R}$, then $f(\lambda)=0$ almost everywhere), then $H_{X}=H_{\xi, \eta}$. In particular, if a process $X$ is Gaussian, then $\eta_{j k}$ and $\xi_{0 k}$ are independent Gaussian random variables.

A result similar to Theorem 3.1 is proved in [16 under extra restrictions that the process $X(t)$ is stationary, the covariance function $R$ of the process is such that

$$
R(\tau) \in L_{1}(\mathbf{R}),
$$

the spectral density is a positive function, and $\widehat{\phi}(y)$ and $\widehat{\psi}(y)$ are functions with finite supports. Theorem 3.1 is proved in [12] without these restrictions. (It also follows from [13.) We prove Theorem 3.1 below together with Remark 3.1 .

In what follows we need the following Karhunen theorem.

Theorem 3.2 (Karhunen [4]). Let $X=\{X(t), t \in T\}$ be a centered second order stochastic process (that is, $\mathrm{E} X(t)=0$ and $\mathrm{E}|X(t)|^{2}<\infty$ for all $t$ ) with the covariance function $R(t, s)=\mathrm{E} X(t) \overline{X(s)}$. Let $\{\Lambda, \mathfrak{A}, \mu\}$ be a measurable space with a $\sigma$-finite measure and let $\{g(t, \lambda), t \in T, \lambda \in \Lambda\}$ be a system of functions such that $g(t, \cdot)$, as a function of the second argument if $t$ is fixed, belongs to the space $L_{2}(\Lambda)$. Then the covariance function $R(t, s)$ is represented in the form

$$
R(t, s)=\int_{\Lambda} g(t, \lambda) \overline{g(s, \lambda)} d \mu(\lambda)
$$

if and only if there exists a random measure $\mathbf{Z}$ with orthogonal values on $\mathfrak{A}$ such that $\mathbf{Z}$ is subordinate to the measure $\mu$ and

$$
X(t)=\int_{\Lambda} g(t, \lambda) d \mathbf{Z}(\lambda)
$$


If the system $\{g(t, \lambda)\}$ is complete in $L_{2}(\Lambda)$, then $H_{X}=H_{\mathbf{Z}}$, where $H_{X}$ is the mean square closure of the sums $\sum_{k=1}^{m} c_{k} X\left(t_{k}\right), t_{k} \in T$, while $H_{\mathbf{Z}}$ is the mean square closure of the sums $\sum_{k=1}^{n} b_{k} \mathbf{Z}\left(S_{k}\right), S_{k} \in \mathfrak{A}$.

Now we turn to the proof of the theorem.

The system $\left\{\phi_{0 k}(x), \psi_{j k}(x), k \in \mathbf{Z}, j=0,1, \ldots\right\}$ is an orthonormal basis in $L_{2}(\mathbf{R})$. The Parseval equality implies that the system

$$
\left\{\frac{1}{\sqrt{2 \pi}} \overline{\widehat{\phi}_{0 k}(y)}, \frac{1}{\sqrt{2 \pi}} \overline{\widehat{\psi}_{j k}(y)}, k \in \mathbf{Z}, j=0,1, \ldots\right\}
$$

also is an orthonormal basis in $L_{2}(\mathbf{R})$. Thus

$$
u(t, y)=\sum_{k \in \mathbf{Z}} a_{0 k}(t) \frac{1}{\sqrt{2 \pi}} \overline{\widehat{\phi}_{0 k}(y)}+\sum_{j=0}^{\infty} \sum_{k \in \mathbf{Z}} b_{j k}(t) \frac{1}{\sqrt{2 \pi}} \overline{\widehat{\psi}_{j k}(y)},
$$

where the series converges in the norm of the space $L_{2}(\mathbf{R})$. Hence

$$
R(t, s)=\int_{\mathbf{R}} u(t, \lambda) \overline{u(s, \lambda)} d \lambda=\sum_{k \in \mathbf{Z}} a_{0 k}(t) \overline{a_{0 k}(s)}+\sum_{j=0}^{\infty} \sum_{k \in \mathbf{Z}} b_{j k}(t) \overline{b_{j k}(s)}
$$

and the series converges for all $t, s \in \mathbf{R}$.

In what follows we consider the space $\{\Lambda, \mathfrak{A}, \mu\}$, where

$$
\Lambda=\{(k, j), k \in \mathbf{Z}, j=0, \ldots, \infty\},
$$

$\mathfrak{A}$ is the family of all subsets of $\Lambda$ and $\mu$ is the measure concentrated at the singletons of the space $\Lambda$ that assigns the weight 1 to each singleton. Now Theorem 3.1 follows from Karhunen's theorem.

Remark 3.1 also follows, since

$$
\left\{a_{0 k}(t), b_{j k}(t), j=0, \ldots, \infty, k \in \mathbf{Z}\right\}
$$

is a complete system in the space $L_{2}(\mathbf{R})$ if and only if the system $\{u(t, \lambda)\}$ is complete in the space $L_{2}(\Lambda)$.

Indeed, the system $W=\left\{a_{0 k}(t), b_{j k}(t), j=0, \ldots, \infty, k \in \mathbf{Z}\right\}$ is complete if the properties

$$
\sum_{k \in \mathbf{Z}}\left|a_{0 k}^{*}\right|^{2}+\sum_{j=0}^{\infty} \sum_{k \in \mathbf{Z}}\left|b_{j k}^{*}\right|^{2}<\infty
$$

and

$$
\sum_{k \in \mathbf{Z}} \overline{a_{0 k}^{*}} a_{0 k}(t)+\sum_{j=0}^{\infty} \sum_{k \in \mathbf{Z}} \overline{b_{j k}^{*}} b_{j k}(t)=0 \quad \text { for all } t \in \mathbf{R}
$$

imply that $a_{0 k}^{*}=0$ and $b_{j k}^{*}=0$.

We have

$$
\begin{aligned}
& \sum_{k \in \mathbf{Z}} \overline{a_{0 k}^{*}} a_{0 k}(t)+\sum_{j=0}^{\infty} \sum_{k \in \mathbf{Z}} \overline{b_{j k}^{*}} b_{j k}(t) \\
& \quad=\frac{1}{\sqrt{2 \pi}} \int_{\mathbf{R}} u(t, y)\left(\sum_{k \in \mathbf{Z}} \overline{a_{0 k}^{*} \widehat{\phi}_{0 k}(y)}+\sum_{j=0}^{\infty} \sum_{k \in \mathbf{Z}} \overline{b_{j k}^{*} \widehat{\psi}_{j k}(y)}\right) d y \\
& \quad=\frac{1}{\sqrt{2 \pi}} \int_{\mathbf{R}} u(t, y) \overline{L(y)} d y=0,
\end{aligned}
$$

where

$$
L(y)=\sum_{k \in \mathbf{Z}} a_{0 k}^{*} \widehat{\phi}_{0 k}(y)+\sum_{j=0}^{\infty} \sum_{k \in \mathbf{Z}} b_{j k}^{*} \widehat{\psi}_{j k}(y)
$$


and $L(y) \in L_{2}(\mathbf{R})$. If the system $\{u(t, y)\}$ is complete, then $L(y)=0$ and thus its Fourier coefficients are zero. If the system $W$ is complete and all $a_{0 k}^{*}$ and $b_{j k}^{*}$ are equal to zero, then $L(y)=0$, that is, the system $\{u(t, \lambda)\}$ is complete.

In what follows a stationary process means a stationary stochastic process in a wide sense.

Corollary 3.1. Let $X=\{X(t), t \in \mathbf{R}\}$ be a centered stationary stochastic process, $R(\tau)=\mathrm{E} X(t+\tau) \overline{X(t)}$. If

1) $R(\tau)$ is a continuous function and the spectral density exists for the process $X(t)$, that is,

$$
R(\tau)=\int_{\mathbf{R}} \exp \{-i \tau \lambda\} f(\lambda) d \lambda
$$

2) $f$ is real-valued, $f(\lambda) \geq 0$, and $\int_{-\infty}^{\infty} f(\lambda) d \lambda=R(0)<\infty$, then

$$
X(t)=\sum_{k \in \mathbf{Z}} \xi_{0 k} \alpha_{0 k}(t)+\sum_{j=0}^{\infty} \sum_{k \in \mathbf{Z}} \eta_{j k} \beta_{j k}(t),
$$

where the series converges in the mean square sense for all $t$, that is,

$$
\begin{gathered}
\sum_{k \in \mathbf{Z}}\left|\alpha_{0 k}(t)\right|^{2}+\sum_{j=0}^{\infty} \sum_{k \in \mathbf{Z}}\left|\beta_{j k}(t)\right|^{2}<\infty, \\
\alpha_{0 k}(t)=\frac{1}{\sqrt{2 \pi}} \int_{\mathbf{R}}(f(y))^{1 / 2} \exp \{-i y(t-k)\} \overline{\widehat{\phi(y)}} d y, \\
\beta_{j k}(t)=\frac{1}{\sqrt{2 \pi} 2^{j / 2}} \int_{\mathbf{R}}(f(y))^{1 / 2} \exp \left\{-i y\left(t-\frac{k}{2^{j}}\right)\right\} \overline{\widehat{\psi}\left(\frac{y}{2^{j}}\right)} d y
\end{gathered}
$$

and where $\xi_{0 k}$ and $\eta_{j k}$ are centered random variables such that

$$
\mathrm{E} \xi_{0 k} \overline{\xi_{0 l}}=\delta_{k l}, \quad \mathrm{E} \eta_{m k} \overline{\eta_{n l}}=\delta_{m n} \delta_{k l}, \quad \mathrm{E} \xi_{0 k} \overline{\eta_{n l}}=0
$$

The following results are obtained in [17] for the random variables $\xi_{0 k}$ and $\eta_{j k}$ appearing in the series (4):

where

$$
\begin{aligned}
\xi_{0 k} & =\int_{\mathbf{R}} X(t) \overline{\Theta_{0 k}(t)} d t, \\
\eta_{j k} & =\int_{\mathbf{R}} X(t) \overline{\gamma_{j k}(t)} d t,
\end{aligned}
$$

$$
\begin{array}{cc}
\widehat{\Theta}_{00}(y)=(\widehat{R}(y))^{-1 / 2} \widehat{\phi}(y), & \Theta_{0 k}(t)=\Theta_{00}(t-k), \\
\widehat{\gamma}_{j 0}(y)=(\widehat{R}(y))^{-1 / 2} \widehat{\psi}_{j 0}(y), \quad \gamma_{j k}(t)=\gamma_{j 0}\left(t-2^{-j} k\right) .
\end{array}
$$

Moreover,

1) $R(\tau) \in L_{1}(\mathbf{R}), R(\tau)$ is continuous, and $\widehat{R}(\tau)>0$ for all $\tau \in \mathbf{R}$,

2) $\widehat{\psi}(y)$ and $\widehat{\phi}(y)$ are functions on $\mathbf{R}$ with finite supports.

\section{Conditions for the uniform Convergence With PRobability one OF SERIES WITH INDEPENDENT TERMS}

Definition 4.1. We say that a continuous function $\sigma=\{\sigma(h), h>0\}$ satisfies condition $\mathbf{A}$ if $\sigma(h)$ increases in the domain $h>0, \sigma(+0)=0$, and

$$
\int_{0}^{\varepsilon}\left|\ln \sigma^{(-1)}(v)\right|^{1 / 2} d v<\infty
$$

for sufficiently small $\varepsilon>0$, where $\sigma^{(-1)}(v)$ is the inverse function to $\sigma(v)$. 
Remark 4.1. Note that condition $\mathbf{A}$ is satisfied for the function

$$
\sigma(h)=\frac{1}{\left(\ln \left(e^{\alpha}+1 / h\right)\right)^{\alpha}}, \quad 0.5<\alpha, \quad h>0 .
$$

The following result is a slight modification of Theorem 3.5.5 of the book 2 .

Lemma 4.1 ([1]). Let $\left\{\xi_{k}, k=1,2, \ldots\right\}$ be a sequence of independent centered random variables with $\mathrm{E}\left|\xi_{k}\right|^{2}=1$ and let $\left\{f_{k}(t), k=1,2, \ldots\right\}$ be a sequence of continuous functions defined on a finite interval $T \subset \mathbf{R}$ and such that

$$
\sum_{k=1}^{\infty} f_{k}^{2}(t)<\infty, \quad t \in T
$$

If a function $\sigma=\{\sigma(h), h>0\}$ satisfies condition $\boldsymbol{A}$ and

$$
\sup _{|t-s| \leq h, t, s \in T}\left|f_{k}(t)-f_{k}(s)\right| \leq b_{k} \sigma(h),
$$

where the sequence $\left\{b_{k}\right\}$ is such that

$$
\sum_{k=1}^{\infty} b_{k}^{2}<\infty
$$

then the series

$$
\sum_{k=1}^{\infty} \xi_{k} f_{k}(t)
$$

converges uniformly in $t \in T$ with probability one.

Lemma 4.1 implies the following result.

Theorem 4.1. Let a stochastic process $X=\{X(t), t \in \mathbf{R}\}$ satisfy the assumptions of Theorem [3.1, let the random variables $\xi_{0 k}$ and $\eta_{j k}, j=0, \ldots, \infty, k \in \mathbf{Z}$, appearing in expansion (3) be independent, and let condition $\boldsymbol{A}$ be satisfied for a function $\sigma=$ $\{\sigma(h), h>0\}$. Then the series (3) converges uniformly in $t \in T$ with probability one on every bounded interval $T \subset \mathbf{R}$ if

$$
\begin{gathered}
\sup _{|t-s| \leq h, t, s \in T}\left|a_{0 k}(t)-a_{0 k}(s)\right| \leq a_{0 k}^{*} \sigma(h), \\
\sup _{|t-s| \leq h, t, s \in T}\left|b_{j k}(t)-b_{j k}(s)\right| \leq b_{j k}^{*} \sigma(h), \\
\sum_{k \in \mathbf{Z}}\left(a_{0 k}^{*}\right)^{2}+\sum_{j=0}^{\infty} \sum_{k \in \mathbf{Z}}\left(b_{j k}^{*}\right)^{2}<\infty
\end{gathered}
$$

for sufficiently small $h$.

Remark 4.2. The random variables $\xi_{0 k}$ and $\eta_{j k}, j=0, \ldots, \infty, k \in \mathbf{Z}$, in expansion (3) are independent if, for example, $X(t)$ is a Gaussian stochastic process.

Lemma 4.2. The following inequality,

$$
v \leq \frac{C_{\alpha, T}}{\left(\ln \left(e^{\alpha}+1 / v\right)\right)^{\alpha}},
$$

holds for $\alpha>0$ and $0<v \leq T$, where the constant $C_{\alpha, T}$ depends on $\alpha$ and $T$ only.

Proof. First we prove inequality (13) for $\alpha \geq 1$.

We check the following bound:

$$
v^{\beta} \leq F_{\gamma, \beta, T} \frac{\gamma^{\gamma}}{\beta^{\gamma}\left(\ln \left(e^{\gamma}+1 / v\right)\right)^{\gamma}}
$$


for $0<\beta \leq 1, \gamma \geq \beta$, and $0<v \leq T$, where $F_{\gamma, \beta, T}$ is a constant depending on $\gamma, \beta$, and $T$ only. Let $u>0$ and $0<s \leq 1$. Then

$$
\ln (1+u) \leq \frac{1}{s} \ln (1+u)^{s} \leq \frac{1}{s} \ln \left(1+u^{s}\right) \leq \frac{u^{s}}{s} .
$$

Setting $u=1 / v, v \geq 0$, in (15) we get

$$
\ln (1+1 / v) \leq 1 /\left(s v^{s}\right) .
$$

Thus

$$
v \leq \frac{1}{(s \ln (1+1 / v))^{1 / s}} .
$$

Raising both sides to the power $\beta>0$ we obtain

$$
v^{\beta} \leq \frac{1}{(s \ln (1+1 / v))^{\beta / s}} .
$$

Put $s=\beta / \gamma$. Then

$$
v^{\beta} \leq \frac{\gamma^{\gamma}}{\beta^{\gamma}(\ln (1+1 / v))^{\gamma}}, \quad v>0, \beta>0, \gamma \geq \beta .
$$

If $0<v \leq T$ and $\gamma>0$, then

$$
\frac{1}{\ln (1+1 / v)} \leq \frac{\widehat{C}_{\gamma, T}}{\ln \left(e^{\gamma}+1 / v\right)},
$$

where

$$
\widehat{C}_{\gamma, T}=\sup _{0<v \leq T} \frac{\ln \left(e^{\gamma}+1 / v\right)}{\ln (1+1 / v)} \leq \sup _{0<v \leq T} \frac{\ln \left(e^{\gamma}(1+1 / v)\right)}{\ln (1+1 / v)} \leq \frac{\gamma}{\ln (1+1 / T)}+1 .
$$

Thus

$$
\frac{1}{\ln (1+1 / v)} \leq \frac{C_{\gamma, T}}{\ln \left(e^{\gamma}+1 / v\right)}
$$

for $0<v \leq T$ and $\gamma>0$, where $C_{\gamma, T}=\gamma / \ln (1+1 / T)+1$.

We obtain inequality (13) for $\alpha \geq 1$ from (14) if $\beta=1$ and $\gamma=\alpha$.

Now we turn to the proof of (13) for $\alpha<1$. If $0<\alpha<1$, then

$$
v \leq \frac{C_{1, T}}{\ln (e+1 / v)} \leq \frac{C_{1, T}}{(\ln (e+1 / v))^{\alpha}} \leq \frac{C_{1, T}}{\left(\ln \left(e^{\alpha}+1 / v\right)\right)^{\alpha}}
$$

for $v \in(0, T]$. This completes the proof of the lemma.

The following result follows from Lemma 4.1 in the paper [10].

Lemma 4.3. Let $\alpha>0, u \geq 0$, and $v>0$. Then

$$
\left|\sin \frac{u}{v}\right| \leq\left(\frac{\ln \left(e^{\alpha}+u\right)}{\ln \left(e^{\alpha}+v\right)}\right)^{\alpha} .
$$

Lemma 4.4. Let $0<a<b$ and let $X=\{X(t), t \in[a, b]\}$ be a stationary process. Let $f$ be the spectral density of $X, g(y):=\sqrt{f(y)}$, $\phi$ be some $f$-wavelet, and let $\psi$ be the corresponding $m$-wavelet. Put $S_{\phi}(y):=\overline{\widehat{\phi}(y)}$ and $S(y):=\overline{\widehat{\psi}(y)}, t_{1}, t_{2} \in[a, b]$.

Assume that the derivatives $g^{\prime}(y), S^{\prime}(y)$, and $S_{\phi}^{\prime}(y)$ exist everywhere and $\left|S^{\prime}(y)\right| \leq M$ for all $y \in \mathbf{R}, g(y)\left|S\left(y / 2^{j}\right)\right| \rightarrow 0$ as $|y| \rightarrow \infty$ for all $j=0,1, \ldots ; g(y)\left|S_{\phi}(y)\right| \rightarrow 0$ as 
$|y| \rightarrow \infty$, and

$$
\begin{gathered}
\int_{\mathbf{R}}|y|\left|g^{\prime}(y)\right|\left(\ln \left(e^{\alpha}+\frac{|y|}{2}\right)\right)^{\alpha} d y<\infty, \\
\int_{\mathbf{R}}|y| g(y)\left(\ln \left(e^{\alpha}+\frac{|y|}{2}\right)\right)^{\alpha} d y<\infty \\
\int_{\mathbf{R}}\left|g^{\prime}(y)\right|\left|S_{\phi}(y)\right|\left(\ln \left(e^{\alpha}+\frac{|y|}{2}\right)\right)^{\alpha} d y<\infty \\
\int_{\mathbf{R}} g(y)\left|S_{\phi}(y)\right|\left(\ln \left(e^{\alpha}+\frac{|y|}{2}\right)\right)^{\alpha} d y<\infty \\
\int_{\mathbf{R}} g(y)\left|S_{\phi}^{\prime}(y)\right|\left(\ln \left(e^{\alpha}+\frac{|y|}{2}\right)\right)^{\alpha} d y<\infty
\end{gathered}
$$

where $\alpha>0$. Then

$$
\sum_{k \in \mathbf{Z}}\left|\alpha_{0 k}\left(t_{1}\right)-\alpha_{0 k}\left(t_{2}\right)\right|^{2}+\sum_{j=0}^{\infty} \sum_{k \in \mathbf{Z}}\left|\beta_{j k}\left(t_{1}\right)-\beta_{j k}\left(t_{2}\right)\right|^{2} \leq \frac{C^{*}}{\left(\ln \left(e^{\alpha}+\frac{1}{\left|t_{2}-t_{1}\right|}\right)\right)^{\alpha}} .
$$

Here the coefficients $\alpha_{0 k}(t)$ and $\beta_{j k}(t)$ are defined by equalities (5) and (6), respectively, and $C^{*}$ is some constant.

Proof. In what follows the symbol $C$ with subscripts and/or superscripts stands for different constants.

Let $k \neq 0$. Integrating equality (6) by parts we get

$$
\begin{aligned}
\sqrt{2 \pi} 2^{j / 2} \beta_{j k}(t)=\frac{i 2^{j}}{k}( & \int_{\mathbf{R}} \exp \left\{i y \frac{k}{2^{j}}\right\} g^{\prime}(y) e^{-i y t} S\left(y / 2^{j}\right) d y \\
& -i t \int_{\mathbf{R}} \exp \left\{i y \frac{k}{2^{j}}\right\} g(y) e^{-i y t} S\left(y / 2^{j}\right) d y \\
& \left.+\frac{1}{2^{j}} \int_{\mathbf{R}} \exp \left\{i y \frac{k}{2^{j}}\right\} g(y) e^{-i y t} S^{\prime}\left(y / 2^{j}\right) d y\right) .
\end{aligned}
$$

Thus

$$
\begin{aligned}
\sqrt{2 \pi} 2^{j / 2}\left|\beta_{j k}\left(t_{1}\right)-\beta_{j k}\left(t_{2}\right)\right| \leq \frac{2^{j}}{|k|}\left(\int_{\mathbf{R}} \mid\right. & g^{\prime}(y) S\left(y / 2^{j}\right)\left(e^{-i y t_{1}}-e^{-i y t_{2}}\right) \mid d y \\
& +\int_{\mathbf{R}} g(y)\left|S\left(y / 2^{j}\right)\right|\left|t_{1} \cdot e^{-i y t_{1}}-t_{2} \cdot e^{-i y t_{2}}\right| d y \\
& \left.+\frac{1}{2^{j}} \int_{\mathbf{R}} g(y)\left|S^{\prime}\left(y / 2^{j}\right)\right|\left|e^{-i y t_{1}}-e^{-i y t_{2}}\right| d y\right) .
\end{aligned}
$$

Let $\alpha$ be an arbitrary positive number. Using the following inequality,

$$
\left|t_{1} \cdot e^{-i y t_{1}}-t_{2} \cdot e^{-i y t_{2}}\right| \leq\left|t_{2}-t_{1}\right|+2 b\left|\sin \frac{y\left(t_{2}-t_{1}\right)}{2}\right|,
$$

and Lemmas 4.2 and 4.3 we get

$$
\sqrt{2 \pi} 2^{j / 2}\left|\beta_{j k}\left(t_{1}\right)-\beta_{j k}\left(t_{2}\right)\right| \leq \frac{M}{|k|} \sigma(|\Delta t|) C_{b}^{(1)},
$$

where

$$
\sigma(|\Delta t|)=\frac{1}{\left(\ln \left(e^{\alpha}+\frac{1}{\left|t_{2}-t_{1}\right|}\right)\right)^{\alpha}}
$$


Therefore

$$
\left|\beta_{j k}\left(t_{1}\right)-\beta_{j k}\left(t_{2}\right)\right| \leq \frac{C_{b}^{(1)} M}{\sqrt{2 \pi} 2^{j / 2}|k|} \sigma(|\Delta t|) .
$$

A similar bound holds for $k \neq 0$ :

$$
\left|\alpha_{0 k}\left(t_{1}\right)-\alpha_{0 k}\left(t_{2}\right)\right| \leq \frac{C_{a}^{(1)}}{\sqrt{2 \pi}|k|} \sigma(|\Delta t|) .
$$

It is clear that

$$
\left|\alpha_{00}\left(t_{1}\right)-\alpha_{00}\left(t_{2}\right)\right| \leq \frac{2}{\sqrt{2 \pi}} \int_{\mathbf{R}} g(y)\left|\sin \frac{y\left(t_{2}-t_{1}\right)}{2}\right|\left|S_{\phi}(y)\right| d y \leq \frac{2 C_{a}^{(2)}}{\sqrt{2 \pi}} \sigma(|\Delta t|) .
$$

Similarly to the proof of a bound for $\left|\beta_{j k}\left(t_{1}\right)-\beta_{j k}\left(t_{2}\right)\right|$, one can show that

$$
\left|\beta_{j 0}\left(t_{1}\right)-\beta_{j 0}\left(t_{2}\right)\right| \leq \frac{2 M C_{b}^{(2)}}{\sqrt{2 \pi} 2^{3 j / 2}} \sigma(|\Delta t|) .
$$

Now the lemma follows from inequalities (19)-(21) and (22).

Theorem 4.2. Let a stationary stochastic process $X=\{X(t), t \in[a, b]\}$ satisfy the assumptions of Corollary 3.1. Assume that the random variables $\xi_{0 k}$ and $\eta_{j k}, j=0, \ldots, \infty$, $k \in \mathbf{Z}$, in expansion (44) are independent. Denote by $f$ the spectral density of the process $X(t)$.

If an $f$-wavelet $\phi$ and the corresponding $m$-wavelet $\psi$ satisfy the assumptions of Lemma 4.4 and $\alpha>0.5$, then the series (4) converges uniformly in $t \in[a, b]$ with probability one.

Proof. The proof follows, since condition A holds for the function $\sigma(h)=\left(\ln \left(e^{\alpha}+1 / h\right)\right)^{-\alpha}$ with $0.5<\alpha$ and one can apply Theorem 4.1 and Lemma 4.4 .

Remark 4.3. An example of a stochastic process and a wavelet satisfying the assumptions of Theorem 4.1 is given by a centered Gaussian stationary process $X=\{X(t), t \in T\}$ with the covariance function $R(\tau)=e^{-\tau^{2}}$ and the Haar wavelet system (assumptions of Lemma 4.4 can easily be checked in this case).

\section{Conditions FOR THE UNIFORM CONVERGENCE IN PROBABILITY OF EXPANSIONS OF STRICTLY $\varphi$-SUB-GAUSSIAN STOCHASTIC PROCESSES}

Let $X=\{X(t), t \in \mathbf{R}\}$ be a strictly $\varphi$-sub-Gaussian stochastic process such that

$$
\mathrm{E} X(t) \overline{X(s)}=R(t, s)=\int_{\mathbf{R}} u(t, \lambda) \overline{u(s, \lambda)} d \lambda,
$$

where $u(t, \lambda), t \in \mathbf{R}$, is a complete function system in the space $L_{2}(\mathbf{R})$. According to Theorem 3.1 and Remark 3.1 the process $X(t)$ is represented in the form of the series (3)), where $\xi_{0 k}$ and $\eta_{j k}$ are uncorrelated $\varphi$-sub-Gaussian random variables such that

$$
\begin{aligned}
& \tau_{\varphi}\left(\xi_{0 k}\right) \leq C_{X}, \\
& \tau_{\varphi}\left(\eta_{j k}\right) \leq C_{X},
\end{aligned}
$$

and where $C_{X}$ is the determining constant of the process $X$.

Definition 5.1. Let $\sigma=\{\sigma(h), h>0\}$ be a continuous increasing function such that $\sigma(+0)=0$. We say that condition $\mathbf{B}$ holds for the function $\sigma$ if

$$
\int_{0}^{\varepsilon} \Psi\left(\ln \frac{1}{\sigma^{(-1)}(u)}\right) d u<\infty
$$


for sufficiently small $\varepsilon>0$, where $\Psi(u)=u / \varphi^{(-1)}(u)$ and $\sigma^{(-1)}(u)$ is the inverse function to $\sigma(u)$.

Theorem 5.1. Let $0<a<b$ and $T=[a, b]$. Assume that

$$
X_{n}=\left\{X_{n}(t), t \in T\right\} \in \operatorname{Sub}_{\varphi}(\Omega)
$$

is a separable stochastic process and that condition $\boldsymbol{B}$ holds for a function

$$
\sigma=\{\sigma(h), h>0\} .
$$

Let

$$
\sup _{|t-s| \leq h} \tau_{\varphi}\left(X_{n}(t)-X_{n}(s)\right) \leq \sigma(h) .
$$

If, for all $t \in T$, the processes $X_{n}(t)$ converge in probability to the process $X(t)$, then $X_{n}(t)$ converge in probability in the space $C(T)$.

Theorem 5.1] is proved in [1] for the space $\mathbf{R}^{k}$.

Now we can prove the following result.

Theorem 5.2. Let a strictly $\varphi$-sub-Gaussian stochastic process $X=\{X(t), t \in \mathbf{R}\}$ have the determining constant $C_{X}$ and satisfy the assumptions of Theorem 3.1. Further assume that a function system $u(t, \lambda)$ is complete in $L_{2}(\mathbf{R})$ and that there exists a function

$$
\sigma_{\varphi}=\left\{\sigma_{\varphi}(h), h>0\right\}
$$

such that condition $\boldsymbol{B}$ holds. If

$$
\begin{gathered}
\sup _{|t-s| \leq h, t, s \in T}\left|a_{0 k}(t)-a_{0 k}(s)\right| \leq a_{0 k}^{*} \sigma_{\varphi}(h), \\
\sup _{|t-s| \leq h, t, s \in T}\left|b_{j k}(t)-b_{j k}(s)\right| \leq b_{j k}^{*} \sigma_{\varphi}(h), \\
\sum_{k \in \mathbf{Z}}\left(a_{0 k}^{*}\right)^{2}+\sum_{j=0}^{\infty} \sum_{k \in \mathbf{Z}}\left(b_{j k}^{*}\right)^{2}<\infty
\end{gathered}
$$

for sufficiently small $h$, then the series (3) converges uniformly in probability on a finite interval $T \subset \mathbf{R}$.

Proof. Theorem 5.2 follows from Theorem 5.1 Indeed put

$$
\begin{gathered}
\widehat{X}(t)=\sum_{k=-N_{0}}^{N_{0}} a_{0 k}(t) \xi_{0 k}+\sum_{j=0}^{N-1} \sum_{k=-M_{j}}^{M_{j}} b_{j k}(t) \eta_{j k}, \\
n=n\left(N_{0}, N, M_{0}, M_{1}, \ldots, M_{N-1}\right)=\min \left\{N_{0}, N, M_{0}, M_{1}, \ldots, M_{N-1}\right\} .
\end{gathered}
$$

Let $|t-s| \leq h$. It is clear that

$$
\begin{aligned}
& \tau_{\varphi}(\widehat{X}(t)-\widehat{X}(s)) \leq C_{X}\left(\mathrm{E}|\widehat{X}(t)-\widehat{X}(s)|^{2}\right)^{1 / 2} \\
& \mathrm{E}|\widehat{X}(t)-\widehat{X}(s)|^{2}=\sum_{k=-N_{0}}^{N_{0}}\left|a_{0 k}(t)-a_{0 k}(s)\right|^{2}+\sum_{j=0}^{N-1} \sum_{k=-M_{j}}^{M_{j}}\left|b_{j k}(t)-b_{j k}(s)\right|^{2} \\
& \leq \sum_{k \in \mathbf{Z}}\left|a_{0 k}(t)-a_{0 k}(s)\right|^{2}+\sum_{j=0}^{\infty} \sum_{k \in \mathbf{Z}}\left|b_{j k}(t)-b_{j k}(s)\right|^{2} \\
& \leq\left(\sum_{k \in \mathbf{Z}}\left(a_{0 k}^{*}\right)^{2}+\sum_{j=0}^{\infty} \sum_{k \in \mathbf{Z}}\left(b_{j k}^{*}\right)^{2}\right) \sigma_{\varphi}(h) .
\end{aligned}
$$


Moreover, for all $t, \widehat{X}(t)$ converges to $X(t)$ in the mean square sense as $n \rightarrow \infty$, whence we obtain the convergence in probability. The theorem is proved.

Corollary 5.1. Let $p \geq 2$,

$$
\alpha>\frac{p-1}{p},
$$

and

$$
\varphi(x)= \begin{cases}\frac{|x|^{p}}{p}, & |x|>1, \\ \frac{x^{2}}{p}, & |x| \leq 1 .\end{cases}
$$

Assume that a stationary strictly $\varphi$-sub-Gaussian stochastic process

$$
X=\{X(t), t \in[a, b]\}
$$

and an $f$-wavelet $\phi$ and the corresponding m-wavelet $\psi$ satisfy the assumptions of Lemma 4.4. Then the series (3) converges uniformly in probability to the process $X(t)$ in the interval $[a, b]$.

Proof. Inequalities (19)-(22) imply that conditions (23)-(25) hold for the functions $\alpha_{0 k}(t)$ and $\beta_{j k}(t)$ involved in expansion (4) of the process $X(t)$, where

$$
\sigma_{\varphi}(h)=\frac{C}{\left(\ln \left(e^{\alpha}+1 / h\right)\right)^{\alpha}}
$$

(here $C$ is some constant). It remains to note that condition $\mathbf{B}$ holds for the function $\sigma_{\varphi}(h)$ if $\alpha>(p-1) / p$.

\section{Concluding Remarks}

We obtained conditions for the uniform convergence of an expansion of a $\varphi$-subGaussian process in a function system generated by wavelets. The rate of convergence will be studied elsewhere.

\section{BIBLIOGRAPHY}

1. L. Beghin, Yu. V. Kozachenko, E. Orsingher, and L. Sakhno, On the solutions of linear oddorder heat-type equations with random initial conditions, J. Stat. Physics 127 (2007), no. 4, 721-739. MR2319850 (2008g:60211)

2. V. V. Buldygin and Yu. V. Kozachenko, Metric Characterization of Random Variables and Random Processes, TViMS, Kiev, 1999; English transl., AMS, Providence, RI, 2000. MR1743716 (2001g:60089)

3. I. Daubechies, Ten Lectures on Wavelets, SIAM, Philadelphia, Pennsylvania, 1992. MR1162107 (93e:42045)

4. I. I. Gikhman, A. V. Skorokhod, and M. I. Yadrenko, Probability Theory and Mathematical Statistics, Vyshcha Shkola, Kiev, 1988. (Russian)

5. R. Giuliano Antonini, Yu. Kozachenko, and T. Nikitina, Spaces of $\phi$-sub-Gaussian random variables, Rendiconti Accademia Nazionale delle Scienze detta dei XL, Memorie di Matematica e Applicazioni, 121 (XXVII) (2003), no. 1, 95-124. MR2056414 (2005f:60036)

6. E. Hernández and G. Weiss, A First Course on Wavelets, CRC Press Inc., Boca Rotan, FL, 1996. MR.1408902 (97i:42015)

7. J.-P. Kahane, Some Random Series of Functions, Lexington, MA, 1968. MR0254888 (40:8095)

8. Yu. V. Kozachenko and Yu. A. Koval'chuk, Boundary value problems with random initial conditions and functional series of $\operatorname{Sub}_{\varphi}(\Omega)$. I, Ukrain. Mat. Zh. 50 (1998), no. 4, 504-515; English transl. in Ukrainian Math. J. 50 (1999), no. 4, 572-585. MR1698149 (2000f:60029)

9. Yu. V. Kozachenko, M. M. Perestyuk, and O. I. Vasylyk, On uniform convergence of wavelet expansions of $\varphi$-sub-Gaussian random processes, Random Oper. Stochastic Equations 14 (2006), no. 3, 209-232. MR.2264363(2008e:60092)

10. Yu. V. Kozachenko and I. V. Rozora, Accuracy and reliability of models of stochastic processes of the space $\operatorname{Sub}_{\varphi}(\Omega)$, Teor. Imovir. Mat. Stat. 71 (2005), 93-104; English transl. in Theory Probab. Math. Statist. 71 (2006), 105-117. MR2144324 (2005m:60077) 
THE UNIFORM CONVERGENCE OF EXPANSIONS OF $\varphi$-SUB-GAUSSIAN PROCESSES

11. Yu. V. Kozachenko and G. I. Slivka, Justification of the Fourier method for hyperbolic equations with random initial conditions, Teor. Imovir. Mat. Stat. 69 (2004), 63-78; English transl. in Theory Probab. Math. Statist. 69 (2005), 67-83. MR2110906 (2005k:60127)

12. Yu. Kozachenko and E. Turchyn, On one Karhunen-Loève-like expansion for stationary random processes, Int. J. Statistics and Management Systems 3 (2008), no. 1-2, 43-55.

13. Yu. V. Kozachenko, I. V. Rozora, and Ye. V. Turchyn, On an expansion of random processes in series, Random Oper. Stochastic Equations 15 (2007), no. 1, 15-33. MR2316186(2008a:60131)

14. Yu. V. Kozachenko, Lectures on Wavelet Analysis, TBiMC, Kyiv, 2004. (Ukrainian)

15. M. A. Krasnosel'skiŭ and Ya. B. Rutickǐ̌, Convex Functions and Orlicz Spaces, Fizmatgiz, Moscow, 1958; English transl., Noordhoff, Groningen, 1961. MR0126722 (23:A4016)

16. G. Walter and J. Zhang, A wavelet-based KL-like expansion for wide-sense stationary random processes, IEEE Trans. Signal Process. 42 (1994), no. 7, 1737-1745.

17. G. Walter and X. Shen, Wavelets and other Orthogonal Systems, Chapman and Hall, CRC, London, 2000. MR1887929 (2003b:42003)

Department of Probability Theory and Mathematical Statistics, Faculty for Mechanics and Mathematics, National Taras Shevchenko University, Academician Glushkov Avenue 6, KYIV 03127, UKRAINE

E-mail address: yvk@univ.kiev.ua

Department of Higher Mathematics, Faculty for Mechanization of Agriculture, DniproPetrovs'k State Agriculture University, Voroshilov Street 25, Dnipropetrovs'K, Ukraine

E-mail address: evgturchyn@ukr.net

Received 17/MAY/2007

Translated by O. I. KLESOV 